\title{
Comparison of Texture Features Based on Gabor Filters
}

\author{
Simona E. Grigorescu, Nicolai Petkov, and Peter Kruizinga
}

\begin{abstract}
Texture features that are based on the local power spectrum obtained by a bank of Gabor filters are compared. The features differ in the type of nonlinear post-processing which is applied to the local power spectrum. The following features are considered: Gabor energy, complex moments, and grating cell operator features. The capability of the corresponding operators to produce distinct feature vector clusters for different textures is compared using two methods: the Fisher criterion and the classification result comparison. Both methods give consistent results. The grating cell operator gives the best discrimination and segmentation results. The texture detection capabilities of the operators and their robustness to nontexture features are also compared. The grating cell operator is the only one that selectively responds only to texture and does not give false response to nontexture features such as object contours.
\end{abstract}

Index Terms-Classification, complex moments, discrimination, features, Fisher criterion, Gabor energy, Gabor filters, grating cells, local power spectrum, segmentation, texture.

\section{INTRODUCTION}

V ARIOUS features related to the local power spectrum of images have been proposed in the literature and used in one way or another for texture analysis, classification, and/or segmentation. In most of these studies the relation to the local spectrum is established through (intermediate) features that are obtained by filtering the input image with a set of two-dimensional (2-D) Gabor filters. Such a filter is linear and local. Its convolution kernel is a product of a Gaussian and a cosine function. The filter is characterized by a preferred orientation and a preferred spatial frequency. Roughly speaking, a 2-D Gabor filter acts as a local band-pass filter with certain optimal joint localization properties in the spatial domain and in the spatial frequency domain [1]. Typically, an image is filtered with a set of Gabor filters of different preferred orientations and spatial frequencies that cover appropriately the spatial frequency domain, and the features obtained form a feature vector field that is further used for analysis, classification, or segmentation.

Gabor feature vectors can be used directly as input to a classification or a segmentation operator or they can first be transformed into new feature vectors that are then used as such an input. In [2]-[8], for example, pairs of Gabor features that correspond to the same preferred orientation and spatial frequency

Manuscript received December 19, 2000; revised May 31, 2002. The associate editor coordinating the review of this manuscript and approving it for publication was Prof. Pierre Moulin.

S. E. Grigorescu and N. Petkov are with the Institute of Mathematics and Computing Science, University of Groningen, Groningen, The Netherlands (e-mail: simona@iwinet.rug.nl; petkov@cs.rug.nl).

P. Kruizinga is with Océ Technologies, Venlo, The Netherlands.

Digital Object Identifier 10.1109/TIP.2002.804262. but differ in the value of a phase parameter are combined in a quantity called the Gabor energy. In references [9], [10], complex moments are derived from Gabor features. Finally, in references [11]-[14] grating cell operator features, inspired by the function of a special type of visual neuron, are computed using Gabor features.

Since the type of "post-Gabor" processing in the above mentioned methods is different, it is interesting to evaluate the effect of the various types of nonlinear post-processing on the usefulness of the resulting features regarding texture discrimination and segmentation.

At this point, the question arises of how to measure the usefulness of different features. Several authors have made a comparison of the performance of various operators and features for texture segmentation. Most of these studies are based on a classification result comparison. In this method, a segmentation algorithm is applied to a feature vector field and the number of misclassified pixels is used to evaluate the segmentation performance and suitability of the features. While this method is widely used for feature comparison [15]-[22], one should keep in mind that it characterizes the joint performance of a feature operator and a subsequent classifier.

In [12] and [13], we proposed a method that can be used to compare the features only, regardless of any subsequent classification or segmentation operations. This method is based on a statistical measure of the capability of a feature operator to discriminate two textures by quantifying the separability of the corresponding clusters of points in the feature space according to the Fisher criterion. While this method of feature evaluation excludes the influence of the classification phase and focuses on the feature extraction operators only, one should be aware of the fact that the choice of a specific evaluation method inevitably introduces certain limitations. In particular, the use of Fisher criterion implies that the mean and the variance of a feature distribution are important and adequate-not necessarily complete-characteristics of the involved distributions, an assumption that has been customarily made in the literature [23]-[30].

None of the aforementioned evaluation methods can be generally considered as superior because each of them is informative in its own way and each has its limitations. Using them both gives a more accurate picture of operators' performance.

This study comes as a natural continuation of the work presented in [13]. There an operator that models the texture processing properties of the visual system of monkeys and, most probably, of man - the grating cell operator-was introduced and compared with other artificial operators that are devised by man. The results showed that the features obtained with the grating cell operator were the best ones for a test image 
material containing oriented textures. These results prompted other questions: is this outcome due only to the orientation selectivity properties of the Gabor filters or is also the type of "post-Gabor" processing that matters; how do nonlinear post-processing schemes devised with mathematical models of the texture in mind perform in comparison with a scheme inspired by the mammal visual system? With this study we try to answer these questions. We restrict the comparison to operators based only on Gabor filters because we evaluated in a similar way other types of operators elsewhere [31]. In this paper, we do not address the issue of Gabor filters selection since this subject has already been sufficiently treated in [32]. Finally, we examine only those types of nonlinear post-processing that were proposed in the literature. Hence, it is beyond the scope of this paper to propose new types of nonlinearities or to combine already proposed ones in order to obtain better features.

The paper is organized as follows: in Section II, we review the linear Gabor filter and various operators based on it. The properties of the concerned operators with respect to texture discrimination are compared in Section III using the Fisher criterion. In Section IV, a number of texture segmentation experiments are carried out and the properties of the considered operators are assessed using the classification result comparison method. In Section $\mathrm{V}$, the robustness of the operators to nontexture input stimuli is studied. The paper is concluded with a discussion in Section VI.

\section{TeXture FeAtures BASED ON GABOR FILTERS}

\section{A. Gabor Filters}

A number of authors used a bank of Gabor filters to extract local image features [2], [4]-[6], [33]. Typically, an input image $I(x, y),(x, y) \in \Omega(\Omega$ - the set of image points $)$, is convolved with a 2-D Gabor function $g(x, y),(x, y) \in \Omega$, to obtain a Gabor feature image $r(x, y)$ as follows:

$$
r(x, y)=\iint_{\Omega} I(\xi, \eta) g(x-\xi, y-\eta) \mathrm{d} \xi \mathrm{d} \eta .
$$

We use the following family of Gabor functions ${ }^{1}$ (for further details we refer to [14] and [34])

$$
g_{\lambda, \Theta, \varphi}(x, y)=e^{-\left(\left(x^{\prime 2}+\gamma^{2} y^{\prime 2}\right) / 2 \sigma^{2}\right)} \cos \left(2 \pi \frac{x^{\prime}}{\lambda}+\varphi\right)
$$

where

$$
\begin{aligned}
x^{\prime} & =x \cos \Theta+y \sin \Theta, \quad y^{\prime}=-x \sin \Theta+y \cos \Theta \\
\sigma & =0.56 \lambda \text { and } \gamma=0.5 .
\end{aligned}
$$

In our experiments, we use two filter banks, one with symmetric $(\varphi=0)$ and the other with antisymmetric $[\varphi=-(1 / 2) \pi]$ Gabor kernels. Each bank comprises 24 Gabor filters that are the result of using three different preferred spatial frequencies of 23,31 , and 47 cycles per image and eight different equidistant preferred orientations $[\Theta=k(\pi / 8), k=0,1, \ldots, 7)$. This

\footnotetext{
${ }^{1}$ Two-dimensional Gabor functions and their power spectra can interactively be generated and visualized at http://www.cs.rug.nl/ imaging/ where a description of the Gabor filter and its relation to a specific type of neuron in the primary
} visual cortex are available as well. type of sampling of the spatial frequency domain takes into account the bandwidth properties of the Gabor filters used [13]. The application of such a filter bank to an input image results in a 24-dimensional feature vector for each point of that image.

\section{B. Gabor Energy Features}

The outputs of a symmetric and an antisymmetric kernel filter in each image point can be combined in a single quantity that is called the Gabor energy. This feature is related to the model of a specific type of orientation selective neuron in the primary visual cortex called the complex cell [35] and is defined in the following way:

$$
e_{\lambda, \Theta}(x, y)=\sqrt{r_{\lambda, \Theta, 0}^{2}(x, y)+r_{\lambda, \Theta,-(1 / 2) \pi}^{2}(x, y)}
$$

where $r_{\lambda, \Theta, 0}(x, y)$ and $r_{\lambda, \Theta,-(1 / 2) \pi}(x, y)$ are the responses of the linear symmetric and antisymmetric Gabor filters, respectively. The result is a new, nonlinear filter bank of 24 channels.

The Gabor energy is closely related to the local power spectrum. The local power spectrum associated with a pixel in an image is defined as the squared modulus of the Fourier transform of the product of the image function and a window function that restricts the Fourier analysis to a neighborhood of the pixel of interest. Using a Gaussian windowing function as the one used in (2) and taking into account (1) and (3) the following relation between the local power spectrum $p_{\lambda, \Theta}$ and the Gabor energy features can be proven:

$$
p_{\lambda, \Theta}(x, y)=e_{\lambda, \Theta}^{2}(x, y)
$$

\section{Complex Moments Features}

In [9] and [36], the real and imaginary parts of the complex moments of the local power spectrum were proposed as features that give information about the presence or absence of dominant texture orientations.

The complex moments of the local power spectrum are defined as follows:

$$
\begin{gathered}
C_{m n}(x, y)=\iint(u+i v)^{m}(u-i v)^{n} \tilde{p}_{u, v}(x, y) \mathrm{d} u \mathrm{~d} v, \\
m, n \in \mathbf{N}
\end{gathered}
$$

where

$$
u=\frac{1}{\lambda} \cos \Theta, \quad v=\frac{1}{\lambda} \sin \Theta, \quad \tilde{p}_{u, v}(x, y)=p_{\lambda, \Theta}(x, y) .
$$

The sum $m+n$, called the order of the complex moment, is related to the number of dominant orientations in the texture. In [36], it is proven that a complex moment of even order $m+n$ has the ability to discriminate textures with $(m+n) / 2$ dominant orientations. More precisely, the moduli of the complex moments give information about the presence or absence of dominant orientations while their arguments specify which orientations are dominant. In [36], the authors discuss the advantages of using the real and imaginary parts of the complex moments as features instead of their moduli and arguments.

In our experiments, we use as features the nonzero real and imaginary parts of the complex moments of the local power 
spectrum. For each point in the image we compute the complex moments of up to order 8 resulting in a set of 45 complex values. From this set we select only the nonzero real and imaginary parts. It can be proven that the complex moments of odd order are zero and that all complex moments $C_{m n}$ for which $m=n$ are real. Moreover, $C_{80}$ and $C_{08}$ are real due to the discretization of the frequency domain used in the computation of the local power spectrum. This amounts to 43 nonzero real values out of which only 24 are linearly independent because $C_{m n}=C_{n m}^{*}$. We use this set of 24 linearly independent values computed for each point in the image as a feature vector associated with that point. In fact, we apply a nonsingular linear transform to the local power spectrum.

We compute the complex moments of up to order 8 in order to obtain the same dimensionality - 24-of the feature space as in the experiments with the other types of feature. Taking only moments of up to order 4 or 6 can be regarded as an implicit feature space dimensionality reduction step. Such a step can improve the separability of the feature clusters, but then this step should also be applied to the other features. Since in the scientific community there is no general agreement whether a space dimensionality reduction step is a part of the feature extraction phase or not, we chose to keep the dimensionality of the feature space the same for all considered operators (see further Section VI).

The local power spectrum features are obtained using the same filter bank as in the computations of the Gabor energy features and consequently have the same coverage of the spatial frequency domain.

\section{Grating Cell Operator Features}

A different type of nonlinearity is applied in an operator that is based on a computational model of a specific type of neuron found in areas V1 and V2 of the visual cortex of macaque monkeys and called the grating cell [37], [38]. Grating cells are selective for orientation but differ from the majority of orientation selective cells found in the mentioned cortical areas in that they do not react to single lines or edges, as for instance simple cells (modeled by Gabor filters) or complex cells (modeled by Gabor energy operators) do. A grating cell only responds when a set of at least three bars of appropriate orientation and spacing is present in its receptive field. The response increases with the number of bars that cross the receptive field of the cell and saturates at about ten bars. The grating cell operator was conceived to reproduce the properties of grating cells as known from electrophysiological researches [11]-[14]. Essentially, this operator signals the presence of one-dimensional (1-D) periodicity of certain preferred spatial frequency and orientation in 2-D images.

The grating cell operator, as proposed in [14], consists of two stages. The first stage is constructed to respond at any position to a set of three parallel bars of a given orientation and spacing at that position. The second stage integrates the output of the first stage in a certain surrounding to ensure that the output of this second stage increases if more than three parallel bars are present in the concerned surrounding. For further details on this operator we refer to [13] and [14].
In our experiments we use a set of grating cell operators with the same eight preferred orientations and three preferred spatial frequencies as in the experiments with the other operators, yielding a vector of 24 features in each point of the image.

\section{Separability of Clusters of Feature Vectors}

In this section the feature extraction operators presented above are compared from the point of view of their ability to discriminate between different textures by means of the Fisher criterion.

\section{A. Comparison Method}

The feature vectors computed in different points of a texture image are not identical; they rather form a cluster in the multidimensional feature space. The larger the distance between two clusters that correspond to two different types of texture, the better the discrimination properties of the texture operator that produced the feature vectors.

In order to determine the distance between two clusters of feature vectors, it is sufficient to look at their projections onto a 1-D space, i.e., a line, under the assumption that this projection maximizes the separability of the clusters in the 1-D space. A linear transform that, under certain conditions, realizes such a projection was first introduced by Fisher [39] and is called the Fisher linear discriminant function. It has the following form:

$$
y=\left(\overrightarrow{\mu_{1}}-\overrightarrow{\mu_{2}}\right)^{T} S^{-1} \vec{x}
$$

where $\overrightarrow{\mu_{1}}$ and $\overrightarrow{\mu_{2}}$ are the means of the two clusters, $S^{-1}$ is the inverse of the pooled covariance matrix of the two clusters, $\vec{x}$ is a feature vector, and $y$ is its 1-D projection.

This projection of the feature vectors into the 1-D space maximizes the Fisher criterion [40], which measures the separability of the two concerned clusters in the reduced space

$$
f=\frac{\left|\eta_{1}-\eta_{2}\right|}{\sqrt{\sigma_{1}^{2}+\sigma_{2}^{2}}}
$$

where $\sigma_{1}$ and $\sigma_{2}$ are the standard deviations of the distributions of the projected feature vectors of the two clusters and $\eta_{1}$ and $\eta_{2}$ are the projections of the means $\overrightarrow{\mu_{1}}$ and $\overrightarrow{\mu_{2}}$, respectively. The Fisher criterion expresses in one single quantity the distance between two clusters relative to their size. The larger the value of the Fisher criterion computed for two clusters, the better the separability of the two clusters.

Strictly speaking, the transform given by (6) need not necessarily maximize the value of $f$ according to (7) for arbitrary distributions. It, however, does so for a Gaussian distribution of texture features, an assumption that has frequently been made and claimed to hold in literature (see e.g., [23]-[30]). In the case of non-Gaussian distributions, one can think of the transform given by (6) as a first order approximation of the transform that maximizes the quantity $f$ in (7).

Widely used in statistics, Fisher criterion has also been used for various purposes in the field of image processing and computer vision: filter design [41], texture classification [21], [42]-[45], and feature space dimensionality reduction [46]. Only recently, this criterion has been applied to the evaluation of texture feature extraction operators [13]. 


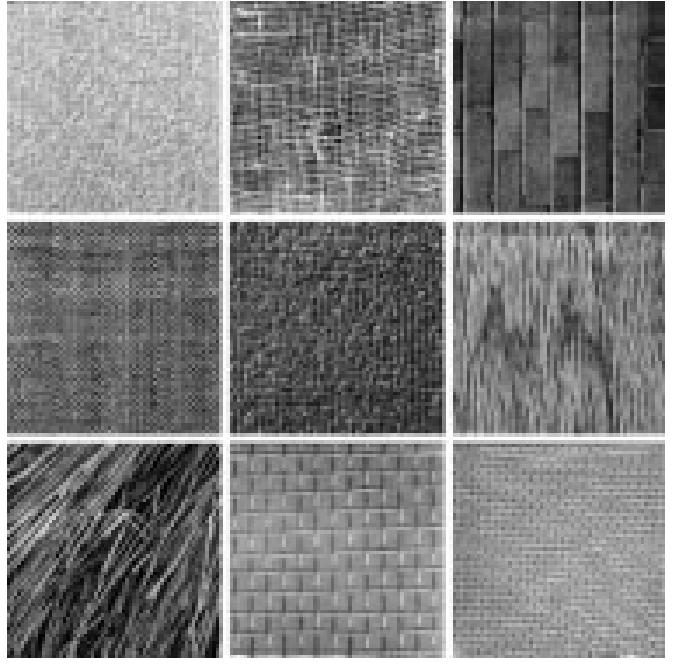

Fig. 1. Nine test images of oriented textures.

\section{B. Results}

We evaluated the performance of the operators presented in Section II according to the Fisher criterion by looking at the pair-wise separability of the feature clusters corresponding to nine test textures (Fig. 1).

While the number of test images used is limited, one has to point out that the only aspect that was taken into account in selecting them is that the textures show a certain degree of "orientedness" which is to guarantee that (some of) the Gabor filters employed will respond. Further, no special attention was paid to selecting these test images and there are no reasons to think that the choice is in favor of any of the feature extraction methods presented above.

The pair-wise separability of the feature clusters corresponding to the nine test textures was measured as follows. The pooled covariance matrix was calculated for each pair of images using 1000 sample feature vectors from each image. Then the feature vectors were projected on a line using the corresponding Fisher linear discriminant function and the Fisher criterion was evaluated in the projection space. For brevity, only essential statistics of the 36 Fisher criterion values computed for each operator are given here (see Fig. 2).

The values obtained for the Gabor energy features are good. The mean value of 6.33 says that there is practically no overlap between two clusters. The worst case scenario, described by the minimum value of 2.35 corresponds to a cluster overlap of less than $2.5 \%$ (assuming Gaussian distribution). The results obtained with the Gabor energy features are remarkable, if one compares them with the linear Gabor features or with the thresholded Gabor features [47], [48]. Our experiments showed that Gabor energy features, involving only a simple type of post-processing, perform better than the linear and thresholded Gabor features by an order of magnitude.

The separability achieved for the complex moments features is smaller than the one achieved with the Gabor energy features. This result is due to the fact that the complex moments were computed from the local power spectrum and not from the Gabor energy features. The nonlinear dependence between the Gabor energy and the local power spectrum (4) leads evidently to different degrees of separability of feature vector clusters. As already re-

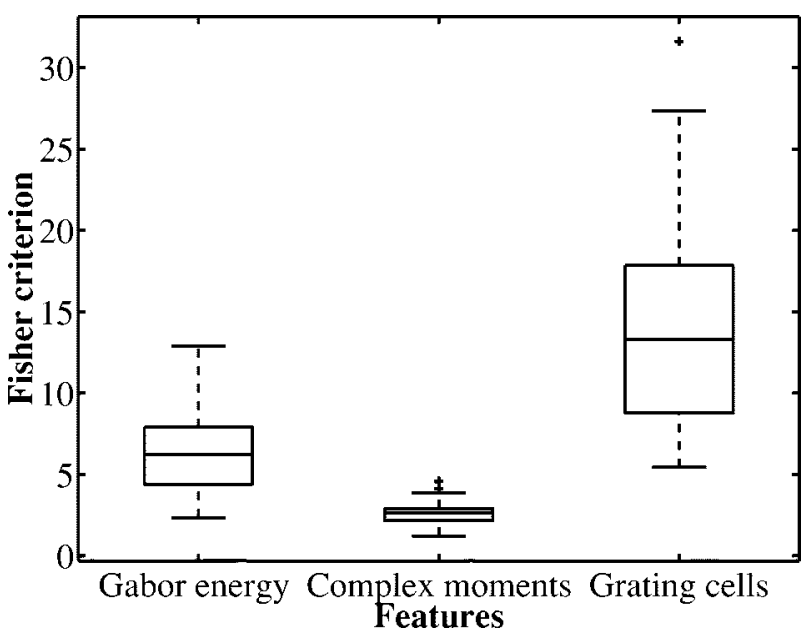

Fig. 2. Boxplot representation of the distribution of the Fisher criterion values obtained with different texture operators.

ported in [31], using the square root in the post-processing phase following the filtering improves the separability of the feature clusters in terms of Fisher criterion. As a possible explanation of this result, let us consider a particular case of two 1-D stochastic variables $X$ and $Y$. For simplicity, we assume that $X=0$ and that $Y$ can take only two values $a$ and $b(0<a<b)$ with equal probability. The value of Fisher criterion in this case is $f_{X, Y}=$ $(a+b) /(b-a)$. Now, if we consider the squares $X^{2}$ and $Y^{2}$ of the two stochastic variables, the value of the Fisher criterion will be $f_{X^{2}, Y^{2}}=\left(a^{2}+b^{2}\right) /\left(b^{2}-a^{2}\right)$ and it can easily be shown that $f_{X, Y}>f_{X^{2}, Y^{2}}$. In other words, the value of the Fisher criterion computed for the two stochastic variables is larger than the value of the Fisher criterion computed for their squares. A similar situation obviously occurs with the features derived from the Gabor energy and its square, the local power spectrum. In this way, features derived directly from the local power spectrum form clusters that are less separable than the clusters obtained with Gabor energy feature vectors.

Computing the complex moments of the local power spectrum can itself not improve the separability of the feature vector clusters obtained from the local power spectrum. As already mentioned in Section II-C above, the computation of the complex moments of the local power spectrum is a nonsingular linear transform of the local power spectrum. Taking in consideration (6) and (7) it can be proven that the value of Fisher criterion is not affected by such a transform.

For any pair of texture images, the inter-cluster distance computed using the grating cell operator features is considerably greater than the inter-cluster distance computed with any of the other operators. The minimum value of the Fisher criterion obtained for this type of feature is 5.44. If (in a first approximation) we assume a Gaussian distribution for the feature clusters, the theoretical cluster overlap corresponding to a Fisher criterion value of 5.44 will be less than $0.01 \%$, corresponding to a misclassification chance of one on ten thousand pixels.

\section{Automatic Texture Segmentation}

In this section, the feature extraction operators presented in Section II are compared in the classical way, i.e., on the basis 


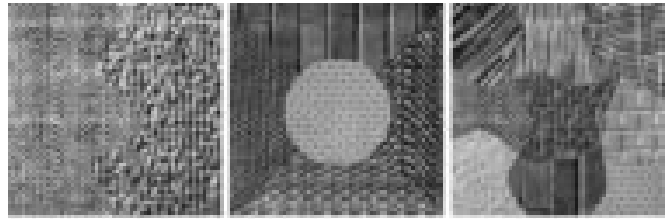

a) Input images

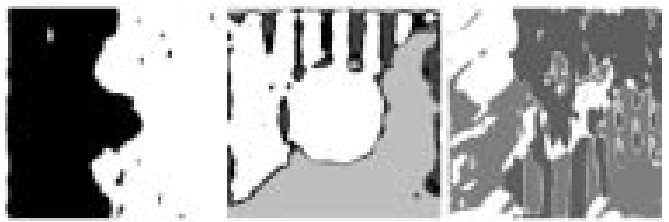

b) Gabor energy features

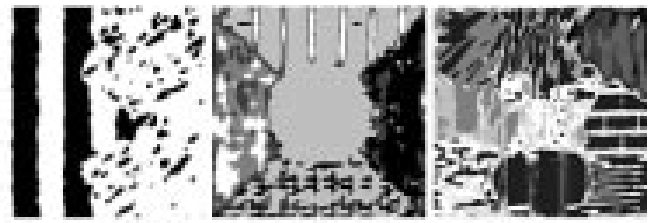

c) Complex moments features
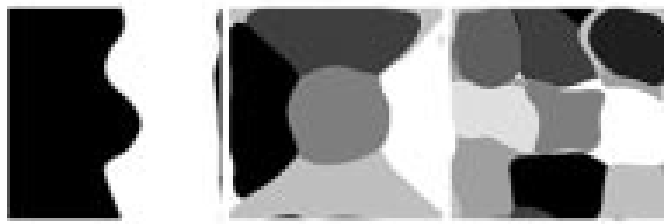

d) Grating cell operator features

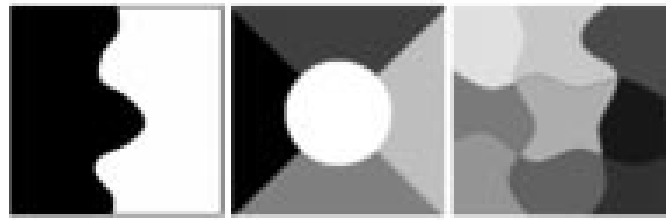

e) Perfect segmentation

Fig. 3. Segmentation results obtained with the fuzzy c-means classification algorithm for texture images containing two, five, and nine oriented textures.

of the segmentation results achieved using a general purpose classifier.

Each operator is applied on three test images containing two, five, and nine textures and its output is fed into a classifier. The test images are presented in Fig. 3(a). These images are constructed out of single texture images so that the perfect segmentation is known [Fig. 3(e)]. The classification is done using the fuzzy c-means clustering algorithm [49]. When working with this algorithm, one has to specify the number of clusters. This number was chosen according to the number of textures in the input image, i.e., it was set to two, five, and nine, respectively.

The segmentation results obtained with the different feature vectors are shown in Fig. 3(b)-(d). In the segmented images, the pixels that correspond to feature vectors of the same cluster are assigned the same gray level. The percentages of correctly classified pixels are given in Fig. 4. Similar results were obtained with the k-means algorithm [50].

The results of the segmentation experiments are in agreement with the results of the Fisher criterion measurements: while the

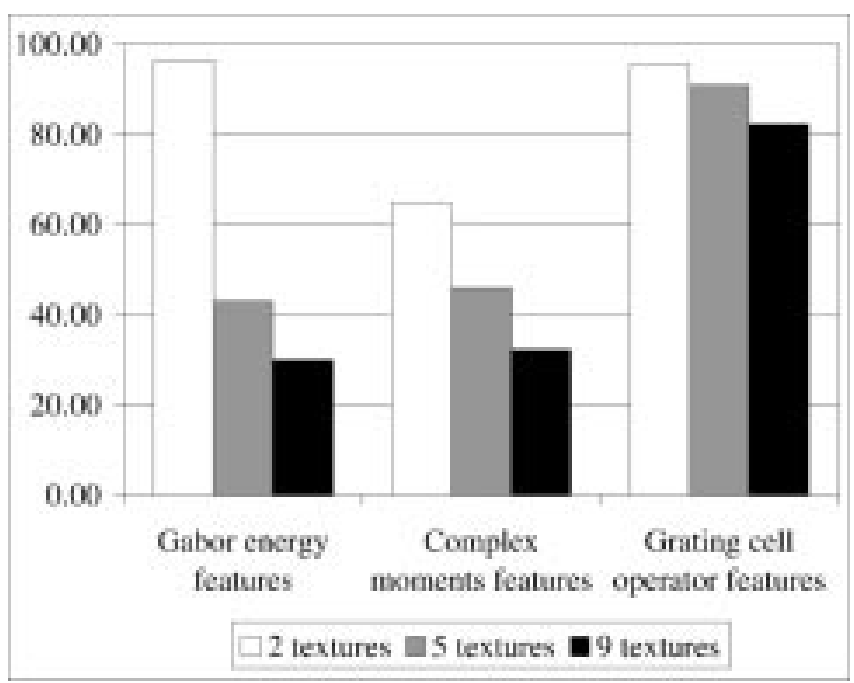

Fig. 4. Percentages of correctly classified pixels using the fuzzy c-means classification algorithm.

Gabor energy leads to a reasonable segmentation, the best segmentation results are achieved with the grating cell operator.

\section{ROBUSTNESS TO NONTEXTURE INPUT}

A minimal requirement on any texture operator is that it is capable of detecting texture at all. In a multichannel filter scheme this means that at least one of the channels must respond or, equivalently, that the $L_{\infty}$-norm of a feature vector $\left(\|\vec{x}\|_{\infty}=\right.$ $\left.\max \left\{\left|x_{i}\right|_{i=1} \ldots n\right\}\right)$ must be different from zero. On one hand, a texture detection operator is thus required to respond to texture. On the other hand, a natural complementary requirement is that such an operator does not respond to every input, more specifically, it does not respond to nontexture input. In this section, we address the robustness of the concerned operators to textured and nontextured inputs.

Fig. 5(a) shows an image of a single object. Disregarding the minor luminance variations of the background and the surface of the object, one can think of this image as containing purely nontexture features. We consider the contours of single objects to be nontexture features. Fig. 5(b)-(d) show the $L_{\infty}$-norm responses of the concerned operators. All operators but the grating cell operator respond to nontexture information and thus falsely detect texture where it is not present.

Fig. 6(a) shows an input image that contains both texture features (in the area occupied by the table cloth) and nontexture features (the contours of the bottle). As illustrated by Fig. 6(b)-(d), all operators respond to the texture features but only the grating cell operator does not respond to nontexture features.

The above remark about a certain class of nontexture features, the contours of single objects, seems to bring a differentiation between different classes of edges and lines: single contour lines and edges, on one hand, being considered as nontexture features versus groups of lines and edges, on the other hand, viewed as texture features. For instance, while the contours of a single leaf of a tree on a plain background are to be seen as nontexture features, the same contours can occur as texture features when they appear in an image together with the contours of many other 


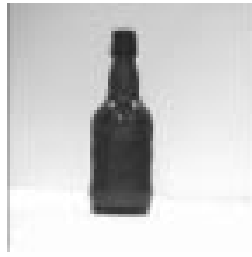

(a)

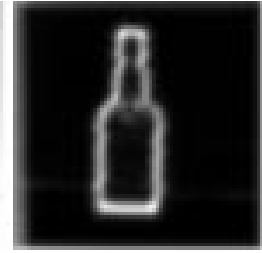

(b)

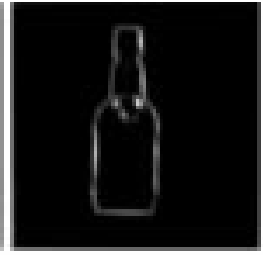

(c)

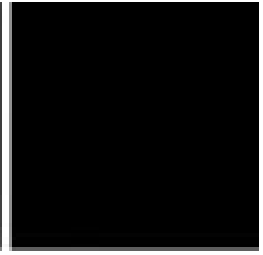

(d)

Fig. 5. (a) Input image containing only nontexture features and the $L_{\infty}$-norm responses of the various operators to this image: (b) Gabor energy operator, (c) complex moments operator, and (d) grating cell operator. All operators but the grating cell operator respond to the nontexture features.

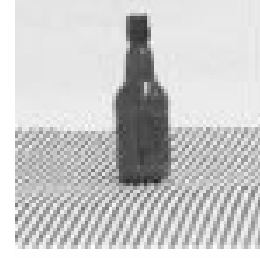

(a)

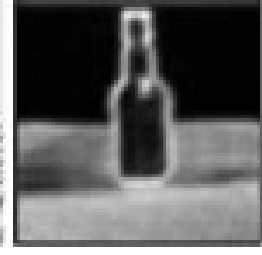

(b)

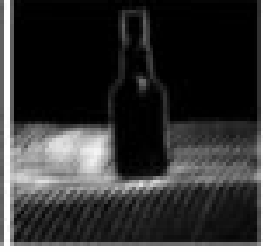

(c)

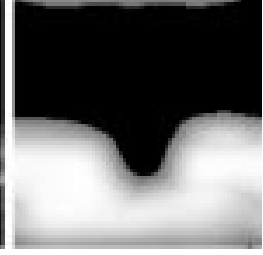

(d)

Fig. 6. (a) Input image containing both texture and nontexture features and the $L_{\infty}$-norm responses of the various operators to this image: (b) Gabor energy operator, (c) complex moments operator, and (d) grating cell operator. Only the grating cell operator shows texture-specific response; the other operators respond to nontexture features as well.

leaves. The use of a separate linguistic entity-the word texture-for a group of edges indicates a semantic difference associated with the context in which an edge appears, stand-alone as a contour of an object or in a group of similar edges forming texture. In a similar way, separate entities have evolved in language to indicate a semantic, context difference between a single tree and a collection of trees-a forest. This differentiation should not be seen as artificial with respect to visual perception because, as various psychophysical experiments have shown, the perception of lines can substantially depend on the presence of other lines in their immediate neighborhood, Fig. 7, see also [51]-[53]. These perceptual differences seem to be mediated by two different types of visual neuron: grating cells, detecting systems of lines [37], [38] versus another type of cell, detecting single lines and edges [38], [54], [55] and called the bar cell [14].

\section{DISCUSSION}

In this paper, we compare a number of texture operators that comprise a Gabor filtering stage followed by different types of nonlinear "post-Gabor" processing. Well aware of the important role of the linear filtering phase for the overall performance of a texture operator [2], [33], [57], in this study, we focus on the performance differences that arise as a consequence of different types of nonlinear post-processing that were used previously by various researchers, particularly in combination with Gabor filters. For this reason, we kept the linear Gabor filtering step the same for all operators. For an analysis of the influence of the sub-band decomposition and the choice of a particular type of linear filter we refer to [32] and [31], respectively.

A possible reduction of the feature space dimensionality is an interesting aspect of any method involving multiple features. For instance, taking a large number of features need not necessarily improve results. On the contrary, it may have a disastrous effect on the performance of the classifier. However, in

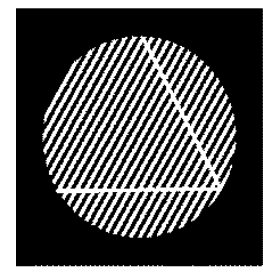

(a)

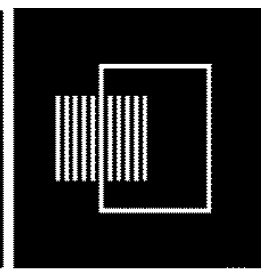

(b)
Fig. 7. Examples of suppression of contour perception by a grating: (a) the side of the triangle that is parallel to the bars of the grating does not pop out as the other two sides do and (b) part of the contour of the rectangle is "lost" in the grating [56].

this paper, we do not treat the problem of feature selection and feature space dimensionality reduction. One reason is that the cluster separability as measured by the Fisher criterion is robust to "the curse of dimensionality": it does not degrade if the number of features is increased. Another reason is that by using a feature space dimensionality reduction step, the values measured in our experiments would represent the joint performance of the post-processing phase and that of the feature space dimensionality reduction step. It is customary in the literature (see for example [46], [58], [59]) to use the classification result comparison method for a performance evaluation of the feature space dimensionality reduction methods. Similarly, Fisher criterion has been used for performance evaluation of a feature selection algorithm [60].

In our experiments, we used textures that show a certain degree of "orientedness" which is to guarantee that (some of) the Gabor filters employed will respond. We did not include textures at different scales and orientations because the operators compared here are not scaling and rotation invariant, a property that is mainly due to the frequency and orientation selectivity of the Gabor filters. The post-processing phase does not compensate for this sensitivity. In the case of complex moments and grating cell operators, it even strengthens this sensitivity. 
As emphasized in [36], the complex moments were designed to react differently to different orientations and different scales. Similarly, in [14], the authors stress that the grating cell operator was conceived to respond only to a given orientation and frequency of the input stimuli. As far as the Gabor energy operator is concerned, the post-processing phase does not eliminate the scale and orientation sensitivity introduced by the filtering phase. If needed, scale and orientation invariance can be added to the methods in a way similar to the one used in other applications [61], [62].

The interclass texture discrimination properties of different features were assessed by Fisher linear discriminant analysis and by the (classical) classification result comparison method. Both approaches gave consistent results. The best results are obtained for the grating cell operator. The complex moments features perform the worst. Interestingly, they perform worse than the Gabor energy features from which they are derived. It turns out to be better to compute complex moments of the Gabor energy than of its square, the local power spectrum. Due to its linearity the complex moments transform itself cannot improve the discrimination effectiveness.

The comparison of the results obtained with grating cell features with those obtained with the other features need a special comment. One should note that the grating cell features are spatially more extended, a property that is due to the weighted averaging step. It was shown [2], [33] that such a post-processing step will in general improve classification or segmentation results. In particular, such a step will make the clusters more compact, leading to improved Fisher criterion values [31]. A question arises then of why this step is included in the grating cell operator but not in the other operators. Our purpose was certainly not to make the grating cell look superior to the other operators. The weighted local averaging part of the grating cell operator is an intrinsic step of the model of a grating cell [14]. It has been included in the model in order to reproduce a specific property of grating cells, namely, that a grating cell starts to respond when at least three parallel bars are present in its receptive field and that its response grows linearly with the addition of further bars to the grating, reaching saturation at about ten bars [37], [38]. In contrast, local averaging was not included in the Gabor energy operator in order to keep this operator as near as possible to its biological counterpart, the complex cell. In a computer vision context—not necessarily biologically motivated-it would of course be interesting to know what the improvement would be if the concerned post-processing step were added to the other operators as well. The effect of such post-processing on various filters was extensively studied in [31]. Indeed, a local averaging post-processing step leads to substantial improvement of the performance of all operators. This improvement is proportional to the performance achieved without that step. With the concerned step, the performance of the Gabor energy operator becomes comparable with that of the grating cell operator and the performance of the complex moment operator improves but remains smaller than that of the other two operators.

Finally, the texture operators were also tested for their ability to detect texture in an image and to separate texture information from other image features like edges and contours of objects.
The grating cell operator is the only one not to give false response to nontexture features such as object edges and to respond specifically to texture features only. The addition of a post-processing step, such as local averaging, cannot change the performance of the other operators in this respect.

\section{REFERENCES}

[1] J. G. Daugman, "Uncertainty relation for resolution in space, spatial frequency, and orientation optimized by two-dimensional visual cortical filters," J. Opt. Soc. Amer. A, vol. 2, no. 7, pp. 1160-1169, 1985.

[2] M. Clark, A. C. Bovik, and W. S. Geisler, "Texture segmentation using Gabor modulation/demodulation," Pattern Recognit. Lett., vol. 6, pp. 261-267, 1987

[3] A. C. Bovik, N. Gopal, T. Emmoth, and A. Restrepo, "Localized measurement of emergent image frequencies by Gabor wavelets," IEEE Trans. Inform. Theory, vol. 38, no. 3, pp. 691-712, 1992.

[4] I. Fogel and D. Sagi, "Gabor filters as texture discriminator," Biol. Cybern., vol. 61, pp. 103-113, 1989.

[5] T. N. Tan, "Texture edge detection by modeling visual cortical channels," Pattern Recognit., vol. 28, no. 9, pp. 1283-1298, 1995.

[6] M. R. Turner, "Texture discrimination by Gabor functions," Biol. Cybern., vol. 55, pp. 71-82, 1986.

[7] B. S. Manjunath and R. Chellappa, "A unified approach to boundary perception: Edges, textures and illusory contours," IEEE Trans. Neural Networks, vol. 4, pp. 96-108, 1993.

[8] W. Y. Ma, "NETRA: A toolbox for navigating large image databases," Ph.D. dissertation, Univ. California, Santa Barbara, 1997.

[9] J. Bigün and J. M. H. du Buf, "N-folded symmetries by complex moments in Gabor space," IEEE Trans. Pattern Anal. Machine Intell., vol. 16, pp. 80-87, Jan. 1994.

[10] P. Schroeter and J. Bigün, "Hierarchical image segmentation by multidimensional clustering and orientation adaptive boundary refinement," Pattern Recognit., vol. 28, no. 5, pp. 695-709, 1995.

[11] P. Kruizinga and N. Petkov, "A computational model of periodic-pattern-selective cells," in Proceedings of the International Workshop on Artificial Neural Networks, J. Mira and F. Sandoval, Eds. Berlin, Germany: Springer-Verlag, 1995, vol. 930, Lecture Notes in Computer Science, pp. 90-99.

[12] — , "Grating cell operator features for oriented texture," in Proc. Int. Conf.Pattern Recognition, A. K. Jain, S. Venkatesh, and B. C. Lovell, Eds., Brisbane, Australia, 1998, pp. 1010-1014.

[13] — - "Non-linear operator for oriented texture," IEEE Trans. Image Processing, vol. 8, pp. 1395-1407, Oct. 1999.

[14] _ - "Computational models of visual neurons specialized in the detection of periodic and aperiodic oriented visual stimuli: Bar and grating cells," Biol. Cybern., vol. 76, pp. 83-96, 1997.

[15] R. W. Conners and C. A. Harlow, "A theoretical comparison of texture algorithms," IEEE Trans. Pattern Anal. Machine Intell., vol. PAMI-2, no. 3, pp. 204-222, 1980.

[16] J. M. H. Du Buf, M. Kardan, and M. Spann, "Texture feature performance for image segmentation," Pattern Recognit., vol. 23, pp. 291-309, 1990.

[17] P. P. Ohanian and R. C. Dubes, "Performance evaluation for four classes of textural features," Pattern Recognit., vol. 25, no. 8, pp. 819-833, 1992.

[18] T. Ojala, M. Pietikainen, and D. Harwood, "A comparative study of texture measures with classification based on feature distributions," Pattern Recognit., vol. 29, no. 1, pp. 51-59, 1996.

[19] O. Pichler, A. Teuner, and B. J. Hosticka, "A comparison of texture feature extraction using adaptive Gabor filtering, pyramidal and tree structured wavelet transforms," Pattern Recognit., vol. 29, no. 5, pp. 733-742, 1996.

[20] K. V. Ramana and B. Ramamoorthy, "Statistical methods to compare the texture features of machined surfaces," Pattern Recognit., vol. 29, no. 9, pp. 1447-1460, 1996.

[21] J. S. Weszka, C. R. Dyer, and A. Rosenfeld, "A comparative study of texture measures for terrain classification," IEEE Trans. Syst., Man, Cybern., vol. SMC-6, pp. 269-285, 1976.

[22] Y. M. Zhu and R. Goutte, "A comparison of bilinear space spatial-frequency representations for texture discrimination," Pattern Recognit. Lett., vol. 16, no. 10, pp. 1057-1068, 1995.

[23] J. M. Jolion, P. Meer, and S. Bataouche, "Robust clustering with applications in computer vision," IEEE Trans. Pattern Anal. Machine Intell., vol. 13, pp. 791-802, Aug. 1991. 
[24] S. Belongie, C. Carson, H. Greenspan, and J. Malik, "Color- and texture-based image segmentation using the expectation-maximization algorithm and its application to content-based image retrieval," in Proc. Int. Conf. Computer Vision, 1998, pp. 675-682.

[25] S. C. Zhu and A. Yuille, "Region competition: Unifying snakes, region growing, and Bayes/MDL for multiband image segmentation," IEEE Trans. Pattern Anal. Machine Intell., vol. 18, pp. 884-900, Sept. 1996.

[26] T. M. Caelli, "Texture classification and segmentation algorithms in man and machine," Spatial Vis., vol. 7, pp. 277-292, 1993.

[27] M. Unser, "Texture classification and segmentation using wavelet frames," IEEE Trans. Image Processing, vol. 4, pp. 1549-1560, Nov. 1995.

[28] T. P. Weldon, W. E. Higgins, and D. F. Dunn, "Gabor filter design for multiple texture segmentation," Opt. Eng., vol. 35, no. 10, pp. 2852-2863, 1996

[29] T. P. Weldon and W. E. Higgins, "Integrated approach to texture segmentation using multiple Gabor filters," in Proc. Int. Conf. Image Processing, 1996, pp. 955-958.

[30] M. Unser, "Local linear transforms for texture measurements," IEEE Trans. Signal Processing, vol. SP-11, pp. 61-79, Jan. 1986.

[31] S. E. Grigorescu, N. Petkov, and P. Kruizinga, "A comparative study of filter based texture operators using Mahalanobis distance," in Proc. Conf. Pattern Recognition, vol. 3, 2000, pp. 897-900.

[32] T. Randen and J. H. Husøy, "Filtering for texture classification: A comparative study," IEEE Trans. Pattern Anal. Machine Intell., vol. 21, pp. 291-310, Apr. 1999.

[33] A. C. Bovik, "Analysis of multichannel narrowband filters for image texture segmentation," IEEE Trans. Signal Processing, vol. 39, pp. 2025-2043, Sept. 1991.

[34] N. Petkov, "Biologically motivated computationally intensive approaches to image pattern recognition," Future Gen. Comput. Syst., vol. 11, pp. 451-465, 1995

[35] H. Spitzer and S. Hochstein, "A complex-cell receptive-field model," $J$ Neurosci., vol. 53, no. 5, pp. 1266-1286, 1985.

[36] J. Bigün and J. M. H. du Buf, "Symmetry interpretation of complex moments and the local power spectrum," Vis. Commun. Image Represent., vol. 6, no. 2, pp. 154-163, 1995.

[37] R. von der Heydt, E. Peterhans, and M. R. Dürsteler, "Grating cells in monkey visual cortex: Coding texture?," in Channels in the Visual Nervous System: Neurophysiology, Psychophysics and Models, B. Blum, Ed. New York: Freund, 1991, pp. 53-73.

[38] ——, "Periodic-pattern-selective cells in monkey visual cortex," J. Neurosci., vol. 12, pp. 1416-1434, 1992.

[39] A. Fisher, The Mathematical Theory of Probabilities. New York: Macmillan, 1923, vol. 1.

[40] K. Fukunaga, Introduction to Statistical Pattern Recognition. New York: Academic, 1990.

[41] T. Randen and J. H. Husøy, "Optimal texture filtering," in Proc. Int. Conf. Image Processing, vol. 1, 1995, pp. 374-377.

[42] T. Y. Philips, A. Rosenfeld, and A. C. Sher, " $O(\log n)$ bimodality analysis," Pattern Recognit., vol. 22, pp. 741-746, 1989.

[43] N. Saito and R. R. Coifman, "Local discriminant bases and their applications," J. Math. Imag. Vis., vol. 5, no. 4, pp. 337-358, 1995.

[44] C. C. Chen and C. L. Huang, "Markov random fields for texture classification," Pattern Recognit. Lett., vol. 14, pp. 907-914, 1993.

[45] S. Allam, M. Adel, and P. Réfrégier, "Fast algorithm for texture discrimination by use of a separable orthonormal decomposition of the co-occurrence matrix," Appl. Opt., vol. 36, no. 32, pp. 8313-8321, 1997.

[46] M. Loog, R. P. W. Duin, and R. Haeb-Umbach, "Multiclass linear dimension reduction by weighted pairwise fisher criteria," IEEE Trans. Pattern Anal. Machine Intell., vol. 23, pp. 762-766, July 2001.

[47] A. K. Jain and F. Farrokhnia, "Unsupervised texture segmentation using Gabor filters," Pattern Recognit., vol. 24, no. 12, pp. 1167-1186, 1991.

[48] J. Malik and P. Perona, "Preattentive texture discrimination with early vision mechanisms," J. Opt. Soc. Amer. A, vol. 7, no. 5, pp. 923-932, 1990.

[49] R. L. Cannon, J. V. Dave, and J. C. Bezdek, "Efficient implementation of the fuzzy c-means clustering algorithms," IEEE Trans. Pattern Anal. Machine Intell., vol. PAMI-8, pp. 248-255, Feb. 1986.

[50] A. Webb, Statistical Pattern Recognition. London, U.K.: E. J. Arnold, 1999.

[51] C. Blakemore, R. H. S. Carpenter, and M. A. Georgeson, "Lateral inhibition between orientation detectors in the human visual system," Nature, no. 228 , pp. $37-39,1970$.
[52] J. J. Knierim and D. C. van Essen, "Neuronal responses to static texture patterns in area V1 of the alert macaque monkey," J. Neurophysiol., vol. 67, pp. 961-980, 1992.

[53] J. A. Solomon and D. G. Pelli, "The visual filter mediating letter identification," Nature, vol. 369, pp. 395-397, 1994.

[54] C. Blakemore and E. A. Tobin, "Lateral inhibition between orientation detectors in the cat's visual cortex," Exper. Brain Res., vol. 15, pp. 439-440, 1972.

[55] P. H. Schiller, B. L. Finlay, and S. F. Volman, "Quantitative studies of single-cell properties in monkey striate cortex. iii. spatial frequencies," J. Neurophysiol., vol. 39, pp. 1334-1351, 1976.

[56] G. Kanizsa, Organization in Vision: Essays on Gestalt Perception. New York: Praeger, 1979

[57] T. Randen and J. H. Husøy, "Texture segmentation using filters with optimized energy separation," IEEE Trans. Image Processing, vol. 8, p. 571, Apr. 1999.

[58] A. K. Jain and D. Zongker, "Feature-selection: Evaluation, application, and small sample performance," IEEE Trans. Pattern Anal. Machine Intell., vol. 19, pp. 153-158, Feb. 1997.

[59] S. de Backer, A. Naud, and P. Scheunders, "Nonlinear dimensionality reduction techniques for unsupervised feature extraction," Pattern Recognit. Lett., vol. 19, no. 8, pp. 711-720, 1998.

[60] M. Kudo and J. Sklansky, "Comparison of algorithms that select features for pattern classifiers," Pattern Recognit., vol. 33, no. 1, pp. 25-41, 2000

[61] J. G. Daugman, "High confidence visual recognition of persons by a test of statistical independence," IEEE Trans. Pattern Anal. Machine Intell., vol. 15, pp. 1148-1161, Nov. 1993

[62] T. Lourens, N. Petkov, and P. Kruizinga, "Large scale natural vision simulations,” Future Gen. Comput. Syst., no. 10, pp. 351-358, 1994.

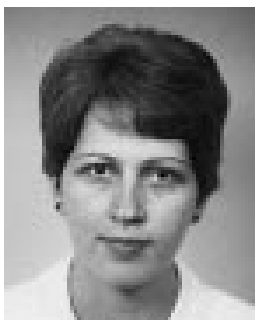

Simona E. Grigorescu received the Dipl.Eng. degree in 1995 and the M.S. degree in 1996 in computer science from Politehnica University, Bucharest, Romania. Since September 1998, she has been pursuing the Ph.D. degree in the Department of Computing Science, University of Groningen, The Netherlands.

From 1996 to 1998, she was Teaching Assistant at the Control and Computer Faculty, Politehnica University. Her areas of research are image processing and pattern recognition.

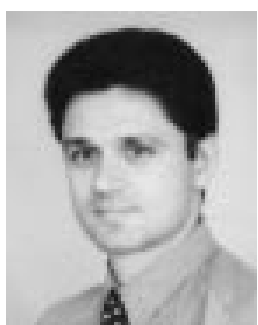

Nicolai Petkov is the Chairman of the Parallel Computing and Intelligent Systems and Scientific Director of the Institute of Mathematics and Computing Science, University of Groningen, The Netherlands. He is author of two books and 60 scientific publications. His current research interests are in the area of computer simulations of the visual system, making links between computer vision, neurophysiology, psychophysics, and arts.

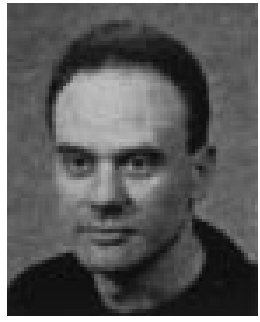

Peter Kruizinga received a M.S. and Ph.D. degrees in computer science from the University of Groningen, The Netherlands, in 1993 and 1999, respectively.

In 2000 he joined Océ Technologies, The Netherlands, where he is currently working on color image processing. His main research interests are image processing, texture analysis and computer models of visual neurons for texture processing. 